\title{
Severe metabolic disorder - cause of death at a newborn suffering from chronic condition
}

\author{
Roxana Tanase ${ }^{1}$, Alina Stoicescu ${ }^{2}$, Elena Georgescu' ${ }^{1}$ Carmen Pascu ${ }^{1}$, Serban Rogoz ${ }^{1}$, \\ Oana Falup-Pecurariu ${ }^{1,3}$ \\ ${ }^{1}$ Brasov Emergency Children's Hospital, Romania \\ ${ }^{2}$ MedLife Brasov Hospital, Romania \\ ${ }^{3}$ Transilvania University Brasov, Romania
}

\begin{abstract}
Congenital adrenal hyperplasia is an autosomal recessive disorder of adrenal steroid biosynthesis, children born from consanguinity relationships have the highest burden of disease.

This is a case report of a 2-month male infant having salt loss deficiency and classic congenital adrenal hyperplasia, without evident signs of virilization. The diagnosis was made based on the medical history of the family, on the clinical and biological data. The outcome can be favorable as long as the glucocorticoid replacement therapy is complied with. Moreover, adrenal crisis and major metabolic imbalance increase the risk of death.

Keywords: congenital adrenal hyperplasia, glucocorticoid, 21-hydroxilase,
\end{abstract} adrenal crisis

\section{INTRODUCTION}

Congenital adrenal hyperplasia (CAH) unites a group of recessively inherited enzyme deficiencies. The most important outcome given by this deficiency refer to modifications occurred in the adrenal steroid production, leading to decreased cortisol and aldosterone and excessive androgens (1). The 21-hydroxilase deficiency represents the most frequent anomaly and accounts for 95\% of $\mathrm{CAH}$ cases (2).

According to recent data, $\mathrm{CAH}$ is classified as follows:

A. Classical form- congenital adrenal hyperplasia

1. 21-hydroxylase deficiency
a) simple virilizing form
b) salt-wasting form
c) slow form (criptica)

2. 11-hydroxylase deficiency-high blood pressure form

3. 3- beta hydroxysteroid dehydrogenase deficiency

4. Deficiency of other steroidogenesis enzymes
B. Non-classical form (with late onset)

1. Virilization and infertility form (latent deficieny of 21-hydroxylase or 3-beta hydroxysteroid dehydrogenase)

2. Virilization and high blood pressure form (latent deficiency of 11-hydroxylase).

The treatment requires a multidisciplinary approach and implies a complex procedure consisting of: steroid replacement, additional salt supplement, avoiding adrenal crisis, surgical correction of external sex organs as well as maintaining an appropriate mental health (1-3).

The screening of all new-born is highly recommended for 21-hydroxylase severe deficiency. Positive tests require further investigations for confirming the diagnosis (2-4).

\section{CASE REPORT}

A male infant, aged 2 months old, is brought to Hospital as he was unable to eat and had psychomotor agitation with inconsolable crying. These symptoms appeared one day before the infant was brought to Hospital. 
From his family history we can observe that the mother aged 26 (GIIPII) and the father aged 33 are 2 nd degree cousins. They have two more offspring, a boy aged 5 and a daughter aged 4 .

The second child, the girl, was previously presented to our Hospital in hypovolemic shock at the age of 6 weeks. Based on the clinical examination and additional investigation, the girl was diagnosed with female pseudo hermaphroditism, salt loss syndrome and adrenogenital disorder. The genotyping assessment confirmed the 21-hydroxylase deficiency. The replacement treatment consisted of Prednisone, Astonin (Fludrocortisone) and additional salt and registered a mild improvement.

The surgical repair of external sex organs was not performed by the age of 4 , because the parents did not comply with the tracking program of this child (Fig. 1).

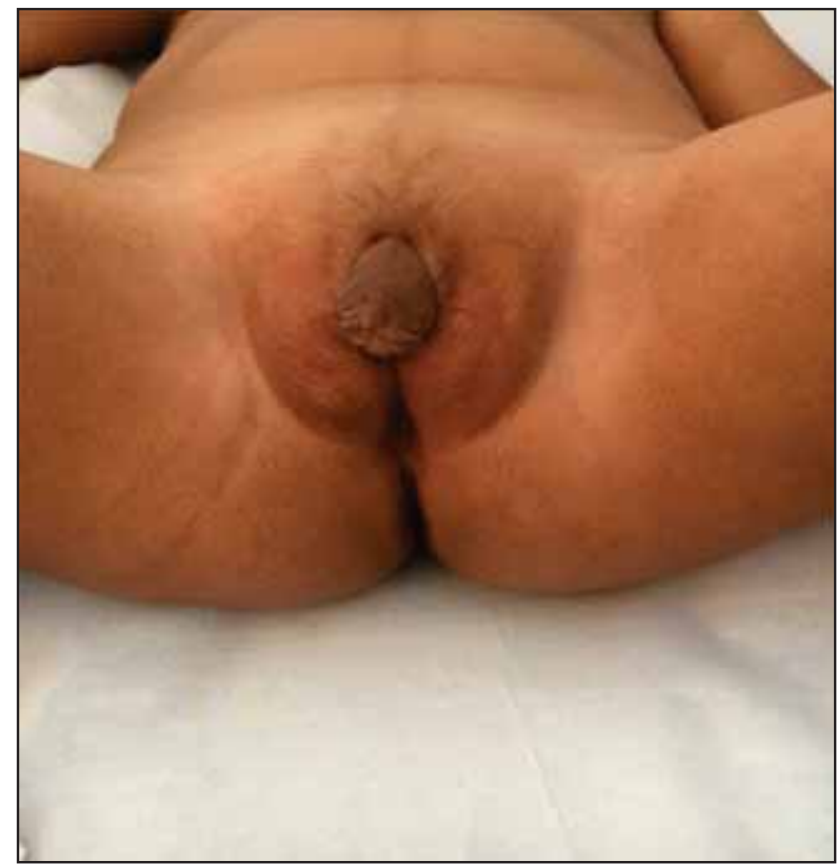

FIGURE 1. 4 year-old girl (our patient's sister) - external genital organs with female pseudohermafroditism and pubic hair.

Our 2-month old patient, the third child of his family, was born out of a pregnancy that was not medically supervised, with physiological evolution. He was delivered at due time, by natural birth, with vertex presentation, birth weight $=3,900 \mathrm{~g}$, mixed feeding, BCG vaccine, uninitiated osteomalacia prevention treatment and weight loss.

The infant was hospitalized in a mediocre general state, $\mathrm{T}=37^{\circ} \mathrm{C}, \mathrm{W}=3,500 \mathrm{~g}$, facial distress, eyes sunk into orbits, dry lips, saburral tongue, faded crying, pale skin, TRC $5 \mathrm{sec}$, cold extremities with cyanosis tendency, normal formed thorax, bi- lateral vesicular murmur, without rales, Sat $\mathrm{O}_{2}$ $98 \%$, AV $150 \mathrm{~b} / \mathrm{min}$, TA 90/60 $\mathrm{mmHg}$, without added breath, supple and abdomen, diminished elasticity skin fold, flabby turgor, semisolid stool, normal diuresis, male external genital organs compliant with age, both testicles descended into scrotum, FA 3/2 cm depressed (Fig. 2).

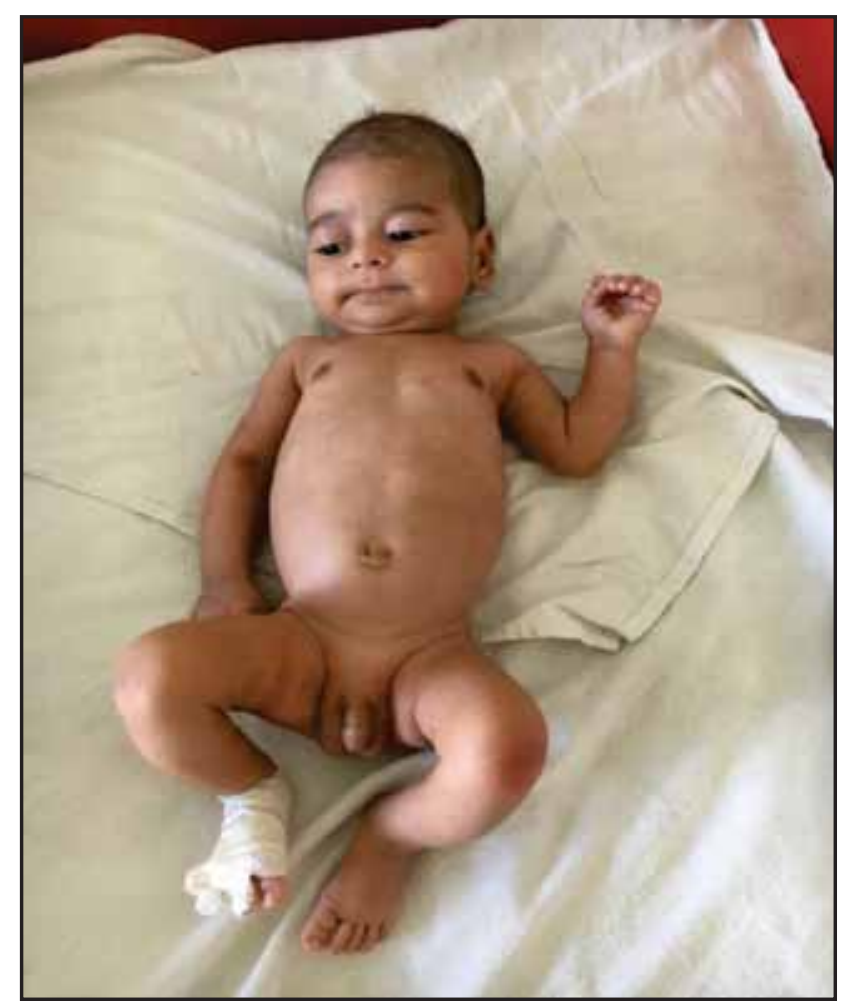

FIGURE 2. Our patient aged 2 months old. IP $=0.64$

Biologically, at the moment of the first hospitalization, we have white blood cells $15,880 / \mathrm{mmc}$, monocytes $11.3 \%$, thrombocytes $690,000 / \mathrm{mmc}$, blood glucose $69 \mathrm{mg} / \mathrm{dl}, \mathrm{Na}=129 \mathrm{mmol} / 1$, ionized calcium $1.68 \mathrm{mmol} / 1$, urea $77.4 \mathrm{mg} / \mathrm{dl}$, potassium $5.55 \mathrm{mmol} / \mathrm{l}$, metabolic acidosis, normal immunogram, urinalysis with leukocytes, positive urine culture with E. coli, stool analysis and culture both negative, negative blood culture, cow milk specific Ig E- negative.

Abdominal and transfontanellar ultrasound without pathological images.

An intravenous therapy was applied tore balance and restore hydration and as nutritional contribution.

Taking into consideration the high risk of this infant to develop an infection, we have initiated the empiric antibiotic therapy with ampicillin $(100 \mathrm{mg} /$ $\mathrm{kg}$ /day), subsequently changed according to the antibiogram. Due to the family medical history of HCS and constant low levels of blood $\mathrm{Na}$ (124-129 $\mathrm{mmol} / \mathrm{l}$ ), we have taken the decision to extend the 


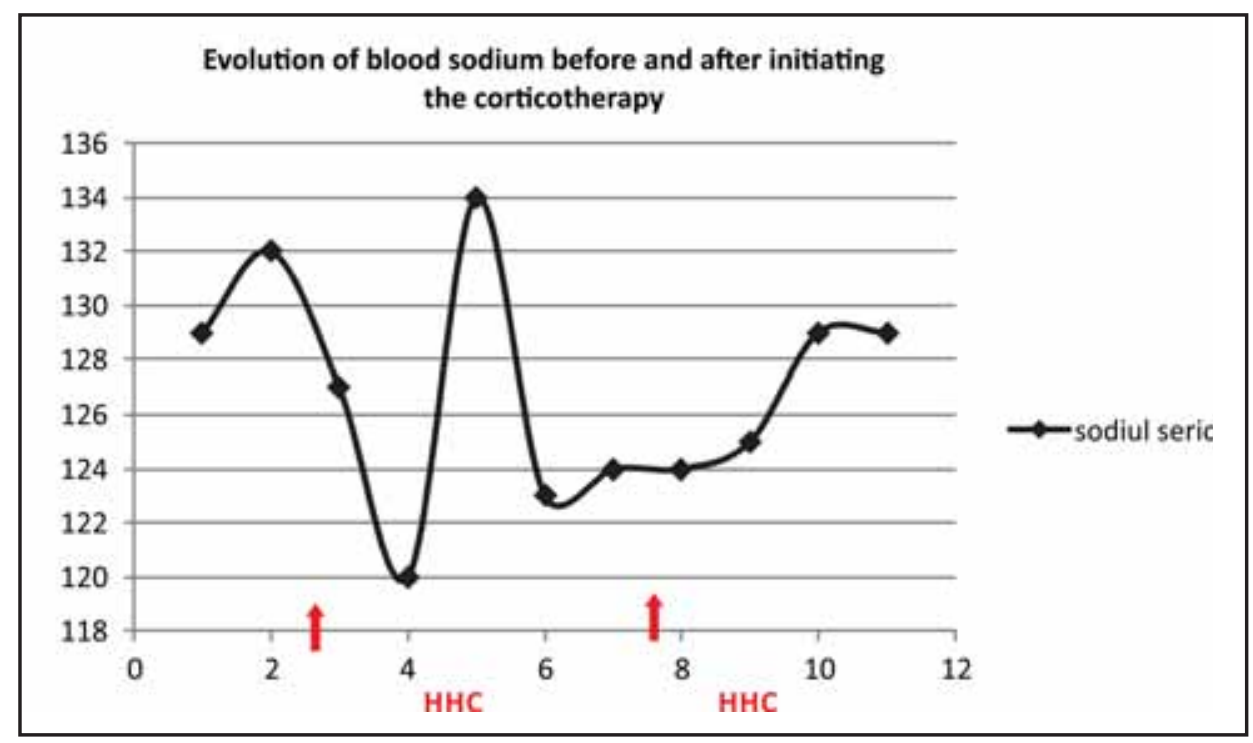

FIGURE 3. Dynamic of the values of blood Na during hospitalization under the hydrocortisone treatment investigations with the following tests: TSH, FT4 (normal values), 17-cetosteroyd/urine $24 \mathrm{H}$ $(\mathrm{x} 2,5 \mathrm{VN} \uparrow), 17$-alfa hidroxyprogesterone, blood cortisol, plasma renin activity $(\mathrm{x} 300 \mathrm{VN} \uparrow)$, aldosterone (normal values), urinary ionogram. The values revealed the diagnosis of adrenogenital syndrome with salt loss. According to the endocrinology clinical guidelines we have added to the treatment scheme the corticotherapy (hydrocortisone) (Fig. 3) and salt supplement until favorable evolution, with return of food appetite and rising weight curve $(\mathrm{G}=3,720 \mathrm{~g}$ after 17 days of substitute treatment).

The genetic consultation is performed and the above mentioned diagnosis is established based on family medical history and on clinical and biological data. The geneticist decided that the confirmation of the CYP21A2 mutation is no longer necessary.

The infant is released at home with substitute treatment with Prednisone $2.5 \mathrm{mg}$ /day po and additional salt in its diet. The infant returns in our care after 3 days, in hypovolemic shock, severe metabolic acidosis, severe hyponatremia $(\mathrm{Na}=123$ $\mathrm{mmol} / \mathrm{l}$ ) and food refusal. The substitution therapy is initialized but the values of sodium continue to be low and heart rhythm disorders appear. Therefore, the sodium therapy is maintained but the patient dies as a result of heart rhythm disorders.

Anatomopathological examination reveals the dimensions and the weight of adrenal glands over the normal values corresponding to the patient length ( $8.3 \mathrm{~g}$ to $5 \mathrm{~g})$. Microscopically we had disorganized architecture of both the adrenal cortices and medullae.

\section{DISCUSSIONS}

Congenital adrenal hyperplasia is a steroid adrenal biosynthesis disorder. Some recessive autosomal forms also affect the gonadal steroidogenesis. 21-hydroxylase, which catalyzes the conversion of 17-hydroxyprogesterone in 11-deoxycortisol, is the most common enzyme deficiency in this pathology. (5).

The mutation of any gene that codified the enzymes used in cortisol or aldosterone synthesis determines congenital adrenal hyperplasia. The particular phenotype that results from these mutations depends upon the gender of the person, the place where the synthesis is blocked and the severity of the mutation (5).

Worldwide incidence of this disorder was estimated at 1:14,199 live births for homozygous patients, 1:60 for heterozygous subjects, with a gene frequency of 0.0083. Incidence of congenital adrenal hyperplasia among whites was estimated to be 1:11,909 for homozygous patients, 1:55 for heterozygous subjects with a gene frequency of 0.0091 . Incidence for the salt-wasting form of congenital adrenal hyperplasia was $1: 18,850$ compared with $1: 57,543$ for congenital adrenal hyperplasia in the simple virilizing form. Thus, salt-wasting congenital adrenal hyperplasia was three times more common than simple virilizing congenital adrenal hyperplasia.

A high prevalence of the disorder was found at the Jewish community from Tunisia and in Eastern Europe, where there is a high rate of consanguineous relationships. Over $50 \%$ of patients are children born out of consanguineous marriages (6). It is observed that our patient is born out of a consanguineous family. 
According to the Clinical Practice Guideline from the Endocrine American Society, universal newborn screening is recommended for severe 21-hydroxylase deficiency and positive results should always be followed up with confirmatory tests $(3,4)$. The screening is controversial because the 17-hydroxyprogesterone levels vary with gestational age and the accuracy of the test can be compromised by cross-reaction with other corticosteroids. In the case of preterm infants there is a high rate of false positive testing and therefore universal screening is not necessary.

There is a high rate of false positive testing at preterms, thus their mass screening is not necessary. Preterms are a specific category of patients that need to be carefully monitored and hospitalized on long periods of time. Any manifestation of the salt-wasting syndrome or adrenal crisis must be immediately observed and promptly treated for this particular category of patients $(7,8)$.

Genotyping should be used only in cases where the diagnosis is equivocal or in the cases where genetic counseling is required. Family data, both clinical and hormone data are the main elements for diagnosis (3), as confirmed by our case study.

Clinically, in classical forms it refers to salt loss syndrome which can lead to life threatening adrenal crisis, due to impossibility to maintain a sodium balance, to female genitalia virilization (clitoris hypertrophy, labial fusion) and to growth stagnation. These symptoms have an early onset, usually under the age of 1 year $(1,9)$. The non-classical forms or with a late onset at a medium age of 5.9 age implies premature pubarche, premature epiphyseal maturation, early growth acceleration and adult short stature, irregular menses, possible infertility at both sexes $(1,9)$, testicular adrenal rest tumors, oligospermia (10). Our patient is suffering from the classical form of the salt loss disease, without virilization sign and exitus by severe metabolic disorder.

The classical treatment consists of replacing cortisol and deficient aldosterone and of managing the symptoms caused by excess androgen by using the lowest possible glucocorticoid dose in order to avoid side effects, especially medication- induced Cushing syndrome $(2,4)$. Hydrocortisone is referred to as the treatment of choice (5). In our case the corticotherapy was initiated with hydrocortisone. The salt was supplemented, according to literature recommendations 2-4 g/day (4).

When the diagnosis is established before birth by genetic tests the treatment with Dexamethasone should be applied in order to prevent the virilization of girls.
During puberty, the cortisol metabolism is modified with an increase in clearance, thus requiring a permanent adjustment of doses, i.e. increasing the dosage. Therapeutically, hydrocortisone can be administered subcutaneous by using a similar technique with the insulin pumps (5).

For girls with severe virilization, single-stage surgical repair should be taken into consideration and should be performed by surgeons experienced in this domain (3). In non-classical forms, the treatment must be adapted according to symptom, its target is represented by maintaining a linear growth curve, normal bone maturation, on time puberty, regular menses, prevention and limitation of the progression of excessive hair and acne. The treatment must be adapted and must not be initiated in order to lower the high hormone concentration (13).

Similar to any other chronicle disease, the impact of the disease and the life-long replacement treatment determines a social isolation of these patients. Therefore, a multi- and inter- disciplinary collaboration between pediatricians, endocrinologists, surgeons, psychologists and geneticists is vital in order to increase their life quality. Besides the fact that the treatment is made on a daily basis, patient with congenital adrenal hyperplasia can have a normal life.

The factors that can affect the quality of life for $\mathrm{CAH}$ patients include lifetime medication, adrenal crisis risk and hyperandrogenic symptoms (12). Patients with $\mathrm{CAH}$ have poor school results and on social plan they have difficulties in establishing friendships with colleagues (14).

In very many cases, the lack of complywith treatment has been reported, thus exposing the patients to adrenal crises. This situation applied in the case of our patient too: the patient's sister presented multiple hospitalizations for hydroelectrolytic imbalance and dehydration syndrome, but the surgical intervention did not take place until the age of 4 due to lack of presentation at the surgery service according to recommendations. Our patient returns from home, after 3 days, with altered general status, severe hyponatremia and acute dehydration.

The replacements treatment schemes are complex and require the administration of medication in certain moments of the day in order to imitate the physiological rhythm of cortisol in the body. Education is a prime element and patients learn how to adjust the doses as response to effort, psychological stress or minor illness. The lack of compliance refers both to the doses prescribed by doctors, as well as to the administration intervals (13). 


\section{CONCLUSIONS}

Consanguinity is a major risk factor in $\mathrm{CAH}$ occurrence. In the above case report, there are two affected children, of different sex, with different evolution at the same disorder. Both presented the classical form of the disorder, namely 21-hydroxilase salt-wasting deficiency, with early diagnosis, at 6 weeks in one case and at 2 months, in the second case. Glucocorticoid therapy replacement and the additional salt supplement were inflicted on both patients, with favorable evolution in the case

\section{REFERENCES}

1. Rachel L. Knowles, Javaria M. Khalid et al. Late clinical presentation of congenital adrenal hyperplasia in older children. Arch Dis Child. 2014; 99(1):30-34.

2. Lisa Nainggolan. Screening for $\mathrm{CAH}$ will save girl's lives, too. The Lancet Diabetes \& Endocrinology February 26, 2013

3. S.C.E. Chapman, S. Llahana et al. Glucocorticoid therapy for adrenal insufficiency. Clin Endocrinol. 2016; 84(5):664-671.

4. Peter C. Hindmars et al. The child difficult to control congenital adrenal hyperplasia. Clin Endocrinol. 2014; 81(1):15-18.

5. Laurie Barclay. Practice guideline recommends universal newborn screening for congenital adrenal hyperplasia. J Clin Endocrinol Metab. 2010; 95:4133-4160.

6. Merke D.P. et al. Aproach to the adult with congenital adrenal hyperplasia due to 21-hydroxylase dediciency. J Clin Endocrinol Metab. 2008; 93(3):653-60 (ISSN:0021-972X).

7. Jean-Claude Carel et al. Don't screen for adrenal hyerplasia in preemies: study. Arcg Pediatr Aolesc Med 2012;166:133-120.

8. Witchel S.F. Nonclassic congenital adrenal hyperplasia. Curr Opin Endocrinol Diabetes Obes. 2012; 19(3):151-8 (ISSN: 1752-2987). of the girl with maintaining a metabolic and hormonal balance and unfavorable in the case of her brother who deceased as a result of adrenal crisis.

\section{Special thanks}

The parents understood the aspects of this pathology and relevant information has been explained to them and they have given their consent in order for the medical information, including pictures, to be used strictly in scientifically and educational purpose. The entire team is thankful for their understanding. Conflict of interest: none declared
Financial support: none declared

9. Marlene Busko. Study rare adrenal disorder tied to gender ambiguity. Medscape medical news 2017 WebMD, LLC.

10. White P.C. et al. Neonatal screening for congenital adrenal hyperplasia. Nat Rev Endocrinol. 2009: 5(9):490-8 (ISSN: 1759-5037).

11. Yau M., Vogiatzi M. et al. Health-related quality of life in children with congenital adrenal hyperplasia. Horm Res Paediatr. 2015; 84(3):16571 (ISSN: 1663-2826).

12. Speisser P.W., Azziz R. et al. Congenital adrenal hyperplasia due to steroid 21-hydroxylase deficiency: an Endocrine Society clinical practice guideline. J Clin Endocrinol Metab. 2010; 95(9): 4133-60 (ISSN: 1945-7197).

13. Vos A.A., Bruinse H.W. Congenital adrenal hyperplasia: do the benefits of prenatal treatment defeat the risk?. Obstet Gynecol Surv. 2010; 65(3):196-205 (ISSN: 1533-9866).

14. Will Boggs. Congenital adrenal hyperplasia diagnosis may be missed until later childhood. Medscape - Oct 11, 2013. 